Collection SFN 10 (2010) 449-463

(C) Owned by the authors, published by EDP Sciences, 2010

DOI: $10.1051 / \mathrm{sfn} / 2010008$

\title{
Les ondes de spin
}

\section{S. Petit}

Institut Rayonnement Matière de Saclay, Laboratoire Léon Brillouin, CEA-CNRS UMR 12, 91190 Gif-sur-Yvette, France

Résumé. Ce cours est consacré à l'étude des ondes de spin. On y expose le formalisme mathématique permettant de calculer leur dispersion ainsi que la fonction de diffusion dans le cas le plus général possible.

\section{INTRODUCTION}

Nous avons déterminé, dans le cours de F. Moussa, l'expression de la section efficace de diffusion magnétique des neutrons :

$$
\begin{aligned}
\frac{\partial^{2} \sigma}{\partial \Omega \partial E^{\prime}}= & \frac{k^{\prime}}{k}\left(\gamma r_{o}\right)^{2} \frac{1}{2 \pi \hbar} \sum_{\ell, \ell^{\prime}, d, d^{\prime}} e^{i \mathbf{Q}\left(\mathbf{R}_{\ell, d^{\prime}}^{o}-\mathbf{R}_{\ell^{\prime}, d^{\prime}}^{o}\right)} F_{d}(\mathbf{Q}) e^{-W_{\ell, d}} F_{d}^{*}(\mathbf{Q}) e^{-W_{\ell^{\prime}, d^{\prime}}} \\
& \times \int d t\left\langle\mathbf{S}_{\perp, \ell, d}(t=0) \cdot \mathbf{S}_{\perp, \ell^{\prime}, d^{\prime}}(t)\right\rangle e^{-i \omega t}
\end{aligned}
$$

où $r_{o}$ désigne la rayon classique de l'électron, $k^{\prime} / k$ traduit le fait que la section efficace est un rapport de flux, $F_{d}(\mathbf{Q})$ désigne le facteur de forme des électrons $d$ non appariés, $W_{\ell, d}$ est le facteur de Debye-Waller dfe l'ion $\ell$.

Par ailleurs, nous avons vu que le magnétisme résulte des corrélations coulombiennes entre électrons du cristal. De surcroît, il s'agit d'un effet de nature quantique, lié à l'antisymétrie des fonctions d'onde. Dans les systèmes où les électrons sont localisés, on le décrit par un Hamiltonien de Heisenberg :

$$
H=\sum_{m, n} J_{m, n} \mathbf{S}_{m} \mathbf{S}_{n}
$$

où $J_{m, n}$ représente l'intégrale d'échange entre les spins $\mathbf{S}_{m}$ et $\mathbf{S}_{n}$.

Le magnétisme est généralement lié à l'existence d'une brisure de symétrie. En effet, dans la plupart des cas, on constate expérimentalement que les spins s'orientent, les uns par rapport aux autres, selon des directions bien précises. Ce choix est dicté par l'apparition, au dessous d'une température critique d'un "champ moléculaire” produit par l'ensemble des spins présents dans le réseau.

Sur le plan statique, ce champ moléculaire induit une nouvelle périodicité dans le cristal, ce qui se traduit typiquement par l'apparition de nouveaux pics de Bragg dans un diagramme de diffraction.

Qu'en est-il du point de vue dynamique ? Comment se traduit l'existence de ce champ moléculaire dans le spectre des excitations ? Pour répondre schématiquement à cette question, il faut se souvenir du mouvement d'un spin dans un champ magnétique. On sait que dans ce cas, le spin précesse autour de la direction du champ avec une fréquence proportionnelle à son intensité. Dans le cristal, par suite du couplage entre spins, ces mouvements de précession ne restent pas localisés, mais se propagent sous forme d'une onde à travers le réseau. On dit souvent qu'il s'agit d'excitations transverses, car le spin tourne dans le plan perpendiculaire à la direction de l'aimantation moyenne.

This is an Open Access article distributed under the terms of the Creative Commons Attribution-Noncommercial License 3.0, which permits unrestricted use, distribution, and reproduction in any noncommercial medium, provided the original work is properly cited. 
Dans la suite de ce cours, nous allons tenter de préciser cette analyse qualitative, en montrant quelles sont ses limites, comment la formaliser, et avec quels outils mathématiques. Nous pourrons alors déterminer la dispersion de ces excitations et par suite déterminer quelles informations on peut déduire d'une expérience de diffusion inélastique des neutrons.

\section{QUELQUES ÉLÉMENTS DE FORMALISME}

\subsection{Le spin en mécanique quantique}

Le spin est un opérateur vectoriel $\mathbf{S}=\left(S^{1}, S^{2}, S^{3}\right)$ dont l'état quantique est représenté par un ket $|\ell\rangle$ d'un espace de Hilbert à $2 S+1$ dimensions. Le nombre quantique $\ell$ represente physiquement la projection du vecteur $\mathbf{S}$ sur l'axe de quantification " $\mathrm{z}$ ", et que l'on appelle $e^{3}$ dans ce cours :

$$
\begin{aligned}
S^{3}|\ell\rangle & =\ell|\ell\rangle, \\
\ell & =-S,-S+1, \ldots, S-1, S .
\end{aligned}
$$

On définit les opérateurs $S^{+}$et $S^{-}$par les relations suivantes :

$$
\begin{aligned}
& S^{+}=S^{1}+i S^{2}, \\
& S^{-}=S^{1}-i S^{2},
\end{aligned}
$$

ainsi que la relation de commutation :

$$
\left[S^{+}, S^{-}\right]=2 S^{3}
$$

Physiquement, ces deux opérateurs augmentent ou diminuent $\ell$ de une unité :

$$
\begin{aligned}
& S^{+}|\ell\rangle=\sqrt{S(S+1)-\ell(\ell+1)}|\ell+1\rangle, \\
& S^{-}|\ell\rangle=\sqrt{S(S+1)-\ell(\ell-1)}|\ell-1\rangle .
\end{aligned}
$$

\subsection{Représentation de Holstein-Primakov}

On élargit cet espace de Hilbert à $2 S+1$ dimensions en introduisant un opérateur boson $b$, dit de Holstein-Primakov, ainsi que l'espace de Fock qui lui est associé. Cet espace est sous-tendu par les kets définis par les nombres d'occupation $n_{b}$ :

$$
\left|n_{b}\right\rangle \text { avec } n_{b}=b^{+} b=0,1,2, \ldots \infty
$$

Définissant le vide $|O\rangle$ comme le ket de $S^{3}$ maximum, on réalise formellement l'identification $|\ell\rangle=\left|S-n_{b}\right\rangle$. On peut alors déterminer l'expression des composantes de spin dans l'espace de Fock :

$$
\begin{aligned}
S^{3} & =S-n_{b}, \\
S^{+} & =\sqrt{2 S-n_{b}} b, \\
S^{-} & =b^{+} \sqrt{2 S-n_{b}} .
\end{aligned}
$$

Mais quelle est donc l'utilité de ce formalisme ? On voit par exemple que cet espace de Fock est bien trop vaste : des états non physiques apparaissent dès que $n_{b} \geq 2 S$, puisque dans ces conditions, la projection du spin sur $e^{3}$ est plus grande que $S$. Cette représentation du spin n'a donc un sens que dans la limite où $n_{b}$ est très faible et donc :

- à basse temperature,

- ou lorsque le spin $S$ est grand (spin "classique").

Ceci étant, l'utilisation de cet espace de Fock a des avantages. Il permet en particulier de mettre à profit l'arsenal mathématique mis au point pour les opérateurs bosons. De plus, en développant $\sqrt{2 S-n_{b}}$ 
en puissance de $n_{b}$, on obtient une description "naturelle" des déviations des spins autour de leur direction privilégiée.

En particulier, en ne gardant que les termes quadratiques dans ces opérateurs, on obtient une approximation du Hamiltonien de Heisenberg. Ce "Hamiltonien d'ondes de spin" a une solution formelle exacte. Celle-ci traduit l'existence de quasi-particules de type boson, appelées magnons ou ondes de spin, et qui ont physiquement le sens de fluctuations "transverses" par rapport à la direction de l'aimantation. Dans la suite de ce cours, nous allons montrer comment calculer leur dispersion. Nous verrons aussi comment calculer les fonctions de corrélation, ce qui permettra de faire le lien avec les expériences de diffusion inélastique des neutrons.

\section{CALCUL DE LA DISPERSION DES ONDES DE SPIN}

On considère par conséquent le Hamiltonien :

$$
H=\sum_{m, n, i, j} \mathbf{S}_{m, i} J_{m, i, n, j} \mathbf{S}_{n, j}
$$

avec

- $m$ un indice qui repère une maille du réseau de Bravais magnétique située à la position $\mathbf{R}_{m}$,

- $i$ un indice qui repère un ion magnétique au sein de cette maille, situé à la position $\mathbf{u}_{i}$,

- enfin, $J_{m, i, n, j}$ décrit l'interaction entre les spins $\mathbf{S}_{m, i}$ et $\mathbf{S}_{n, j}$ situés aux positions $\mathbf{R}_{m}+\mathbf{u}_{i}$ et $\mathbf{R}_{n}+\mathbf{u}_{j}$. Dans le cas général, il s'agit d'une matrice $3 \times 3$ dont les éléments sont les $J_{m, i, n, j}^{\alpha \beta}$. On suppose en outre que ces derniers ne dépendent que de la distance entre ions.

\subsection{Traitement des anisotropies}

Les termes d'anisotropie planaire ou uniaxiale peuvent être absorbés dans la définition de $J_{m, i, n, j}$. En effet alors que l'échange "classique" est associé aux termes $i \neq j$, les termes d'anistropie vont correspondre aux termes du type $m=n, i=j$. Par exemple, pour prendre en compte un terme traduisant l'existence d'un plan de facile aimantation, ici le plan $x y$ :

$$
H_{D}=D \sum_{m, i} S_{m, i}^{z} S_{m, i}^{z} \quad \text { avec } D \geq 0
$$

on posera : $J_{m, i, m, i}^{z z}=D$.

\subsection{Hypothèses de base}

1. L'hypothèse fondamentale de l'approximation des ondes de spin consiste à supposer que le champ moléculaire stabilise une structure magnétique ordonnée. Dans la suite, nous allons supposer que cette structure définit un réseau magnétique qui reste commensurable avec le réseau atomique. Chaque maille $m$ de ce réseau magnétique est alors constituée d'un motif comportant $L$ ions. Pour chacun, on peut définir une base locale privilégiée $\left(e_{i}^{1}, e_{i}^{2}, e_{i}^{3}\right)$ avec $e_{i}^{3}=e_{i}^{1} \times e_{i}^{2}$, et dans laquelle :

$$
\mathbf{S}_{m, i}=\left(S_{m, i}^{1}, S_{m, i}^{2}, S_{m, i}^{3}\right) \quad \text { avec }\left\langle S_{m, i}^{1}\right\rangle=\left\langle S_{m, i}^{2}\right\rangle=0,\left\langle S_{m, i}^{3}\right\rangle \neq 0 .
$$

Ces bases individuelles locales sont reliées à la base de l'espace cartésien ordinaire $(x, y, z)$ par une matrice de passage $R_{i}^{\alpha, \mu}$ :

$$
S_{i}^{\alpha}=\sum_{\mu=1,2,3} R_{i}^{\alpha, \mu} S_{i}^{\mu}
$$


2. La deuxième hypothèse consiste à utiliser la représentation de Holstein-Primakov, en introduisant un opérateur boson $b_{m, i}$ par maille $m$ et par ion $i$ du motif. En développant à l'ordre le plus bas dans l'hypothèse où $n_{b} \leq 2 S$, on obtient les expressions suivantes pour les composantes de spin :

$$
\begin{aligned}
& S_{m, i}^{+} \approx \sqrt{2 S_{i}} b_{m, i} \\
& S_{m, i}^{1} \approx \frac{\sqrt{2 S_{i}}}{2}\left(b_{m, i}+b_{m, i}^{+}\right) \\
& S_{m, i}^{-} \approx b_{m, i}^{+} \sqrt{2 S_{i}} \\
& \text { et } S_{m, i}^{2} \approx \frac{\sqrt{2 S_{i}}}{2 i}\left(b_{m, i}-b_{m, i}^{+}\right) \\
& S_{m, i}^{3} \approx S_{i}-b_{m, i}^{+} b_{m, i} .
\end{aligned}
$$

En définissant les vecteurs $\mathbf{z}$ et $\eta$ par leurs composantes :

$$
\begin{aligned}
z_{i}^{\alpha} & =R_{i}^{\alpha, 1}+i R_{i}^{\alpha, 2} \\
\eta_{i}^{\alpha} & =R_{i}^{\alpha, 3}
\end{aligned}
$$

on trouve pour les composantes du spin dans la base cartésienne :

$$
S_{m, i}^{\alpha=x, y, z}=\frac{\sqrt{2 S_{i}}}{2} \bar{z}_{i}^{\alpha} b_{m, i}+\frac{\sqrt{2 S_{i}}}{2} z_{i}^{\alpha} b_{m, i}^{+}+\eta_{i}^{\alpha}\left(S_{i}-b_{m, i}^{+} b_{m, i}\right) .
$$

\section{Remarque :}

Dans le cas où le réseau magnétique n'est pas commensurable avec le réseau nucléaire, la matrice de changement de base $R$ de même que les vecteurs $\mathbf{z}$ et $\eta$ dépendent de l'indice $m$.

\subsection{Le Hamiltonien d'ondes de spin}

Compte tenu des deux hypothèses fondamentales, le Hamiltonien de Heisenberg devient le Hamiltonien d'ondes de spin et s'écrit

$$
\begin{aligned}
H= & \sum_{m, n, i, j, \alpha, \beta}\left\{\frac{\sqrt{2 S_{i}}}{2} \bar{z}_{i}^{\alpha} b_{m, i}+\frac{\sqrt{2 S_{i}}}{2} z_{i}^{\alpha} b_{m, i}^{+}+\eta_{i}^{\alpha}\left(S_{i}-b_{m, i}^{+} b_{m, i}\right)\right\} J_{m, i, n, j}^{\alpha \beta} \\
& \times\left\{\frac{\sqrt{2 S_{j}}}{2} \bar{z}_{j}^{\beta} b_{n, j}+\frac{\sqrt{2 S_{j}}}{2} z_{j}^{\beta} b_{n, j}^{+}+\eta_{j}^{\beta}\left(S_{j}-b_{n, j}^{+} b_{n, j}\right)\right\} .
\end{aligned}
$$

En définissant les vecteurs colonnes $X_{m}$ :

$$
X_{m}=\left(\begin{array}{c}
b_{m, 1} \\
\ldots \\
b_{m, i} \\
\ldots \\
b_{m, L} \\
b_{m, 1}^{+} \\
\ldots \\
b_{m, i}^{+} \\
\ldots \\
b_{m, L}^{+}
\end{array}\right)
$$

on obtient finalement :

$$
H=\sum_{m, n} X_{m}^{+} h_{m, n} X_{n}
$$


où $h_{m, n}$ est une matrice par blocs de dimension $2 L \times 2 L$ dont les éléments $h_{m, n, i, j}$ sont donnés par:

$$
\left(\begin{array}{ccc}
-\Omega_{m, n, i, j}+\frac{\sqrt{S_{i} S_{j}}}{2} \mathbf{z}_{i} J_{m, i, n, j} \overline{\mathbf{z}}_{j} & +\frac{\sqrt{S_{i} S_{j}}}{2} \mathbf{z}_{i} J_{m, i, n, j} \mathbf{z}_{j} \\
+\frac{\sqrt{S_{i} S_{j}}}{2} \overline{\mathbf{z}}_{i} J_{m, i, n, j} \overline{\mathbf{z}}_{j} & -\Omega_{m, n, i, j}+\frac{\sqrt{S_{i} S_{j}}}{2} \overline{\mathbf{z}}_{i} J_{m, i, n, j} \mathbf{z}_{j}
\end{array}\right)
$$

et

$$
\Omega_{m, n, i, j}=\delta_{m, n} \delta_{i, j}\left(\sum_{\nu, \ell} S_{\ell} \eta_{i} J_{m, i, v, \ell} \eta_{\ell}\right)
$$

\subsection{Transformation de Fourier}

Ce Hamiltonien quadratique peut être partiellement diagonalisé à l'aide de la transformation de Fourier. En effet, $h_{m, n}$ ne dépend que de la différence $\mathbf{R}_{v}=\mathbf{R}_{n}-\mathbf{R}_{m}$. On pose ( $N$ est le nombre de mailles élémentaires) :

$$
\begin{aligned}
& X_{m}=\frac{1}{\sqrt{N}} \sum_{\mathbf{k}} X_{\mathbf{k}} e^{i \mathbf{k} \mathbf{R}_{m}}, \\
& X_{m}^{+}=\frac{1}{\sqrt{N}} \sum_{\mathbf{k}} X_{\mathbf{k}}^{+} e^{-i \mathbf{k} \mathbf{R}_{m}} .
\end{aligned}
$$

En introduisant le nouvel opérateur

$$
b_{\mathbf{k}, i}=\frac{1}{\sqrt{N}} \sum_{m} b_{m, i} e^{-i \mathbf{k} \mathbf{R}_{m}}
$$

ainsi que son adjoint $b_{\mathbf{k}, i}^{+}$, on montre que :

$$
X_{\mathbf{k}}=\left(\begin{array}{c}
b_{\mathbf{k}, 1} \\
\ldots \\
b_{\mathbf{k}, i} \\
\ldots \\
b_{\mathbf{k}, L} \\
b_{-\mathbf{k}, 1}^{+} \\
\cdots \\
b_{-\mathbf{k}, i}^{+} \\
\cdots \\
b_{-\mathbf{k}, L}^{+}
\end{array}\right)
$$

Le vecteur $k$ est ici un vecteur du réseau réciproque associé au réseau de Bravais magnétique. On obtient donc :

$$
\begin{aligned}
& H=\sum_{\mathbf{k}, \mathbf{k}^{\prime}} \frac{1}{N} X_{\mathbf{k}}^{+}\left(\sum_{m, n} h_{m, n} e^{-i \mathbf{k} \mathbf{R}_{m}+i \mathbf{k}^{\prime} \mathbf{R}_{n}}\right) X_{\mathbf{k}^{\prime}} \\
& =\sum_{\mathbf{k}, \mathbf{k}^{\prime}} \frac{1}{N} X_{\mathbf{k}}^{+}\left(\sum_{m}-i\left(\mathbf{k}-\mathbf{k}^{\prime}\right) \mathbf{R}_{m} \sum_{v} h_{v} e^{i \mathbf{k}^{\prime} \mathbf{R}_{v}}\right) X_{\mathbf{k}^{\prime}}
\end{aligned}
$$




$$
\begin{aligned}
& =\sum_{\mathbf{k}, \mathbf{k}^{\prime}} \frac{1}{N} X_{\mathbf{k}}^{+}\left(N \delta_{\mathbf{k}, \mathbf{k}^{\prime}} \sum_{\nu} h_{v} e^{i \mathbf{k}^{\prime} \mathbf{R}_{v}}\right) X_{\mathbf{k}^{\prime}} \\
& =\sum_{\mathbf{k}} X_{\mathbf{k}}^{+}\left(\sum_{v} h_{v} e^{i \mathbf{k} \mathbf{R}_{v}}\right) X_{\mathbf{k}} .
\end{aligned}
$$

et donc :

$$
H=\sum_{\mathbf{k}} X_{\mathbf{k}}^{+} h_{\mathbf{k}} X_{\mathbf{k}}
$$

On fait ainsi apparaître la transformée de Fourier de l'interaction d'échange, définie par :

$$
J_{\mathbf{k}, i, j}=\sum_{\nu} J_{v, i, j} e^{i \mathbf{k} \mathbf{R}_{v}}
$$

Comme $J_{m, n, i, j}=J_{n, m, j, i}$, on notera les propriétés remarquables suivantes :

$$
J_{\mathbf{k}, i, j}^{*}=J_{-\mathbf{k}, i, j}=J_{\mathbf{k}, j, i}
$$

La matrice $h_{\mathbf{k}}$ est de dimension $2 L \times 2 L$, et ses éléments de matrice $h_{\mathbf{k}, i, j}$ sont donnés par :

et

$$
\left(\begin{array}{ccc}
-\Omega_{\mathbf{k}, i, j}+\frac{\sqrt{S_{i} S_{j}}}{2} \mathbf{z}_{i} J_{\mathbf{k}, i, j} \overline{\mathbf{z}}_{j} & +\frac{\sqrt{S_{i} S_{j}}}{2} \mathbf{z}_{i} J_{\mathbf{k}, i, j} \mathbf{z}_{j} \\
+\frac{\sqrt{S_{i} S_{j}}}{2} \overline{\mathbf{z}}_{i} J_{\mathbf{k}, i, j} \overline{\mathbf{z}}_{j} & -\Omega_{\mathbf{k}, i, j}+\frac{\sqrt{S_{i} S_{j}}}{2} \overline{\mathbf{z}}_{i} J_{\mathbf{k}, i, j} \mathbf{z}_{j}
\end{array}\right) .
$$

$$
\Omega_{\mathbf{k}, i, j}=\delta_{i, j}\left(\sum_{\ell} S_{\ell} \eta_{i} J_{k=0, i, \ell} \eta_{\ell}\right)
$$

\subsection{Transformation de Bogolubov}

Du fait de cette structure par blocs, il reste, pour achever la diagonalisation du Hamiltonien, à déterminer pour chaque valeur de $k, L$ opérateurs bosons $y_{\mathbf{k}, \ell}$ et leurs adjoints $y_{\mathbf{k}, \ell}^{+}$, définis de telle sorte que $H$ prenne dans cette nouvelle "base" une forme diagonale. Plus exactement, on définit le vecteur colonne $Y_{\mathbf{k}}=\left\{. . y_{\mathbf{k}, \ell} \ldots, . . y_{-\mathbf{k}, \ell}^{+} \ldots\right\}$ comme une combinaison linéaire des opérateurs $X_{\mathbf{k}}$ :

$$
X_{\mathbf{k}}=P_{\mathbf{k}} Y_{\mathbf{k}}
$$

où la matrice $P_{\mathbf{k}}$ doit être choisie de telle sorte que $P_{\mathbf{k}}^{+} h_{\mathbf{k}} P_{\mathbf{k}}=E_{\mathbf{k}}$ avec $E_{\mathbf{k}}$ une matrice diagonale :

$$
\begin{aligned}
H & =\sum_{\mathbf{k}} X_{\mathbf{k}}^{+} h_{\mathbf{k}} X_{\mathbf{k}}=\sum_{\mathbf{k}} Y_{\mathbf{k}}^{+} E_{\mathbf{k}} Y_{\mathbf{k}} \\
& =\sum_{\mathbf{k}, \ell=1, \ldots, L} E_{\mathbf{k}, \ell} y_{\mathbf{k}, \ell}^{+} y_{\mathbf{k}, \ell}+E_{\mathbf{k}, \ell+L} y_{-\mathbf{k}, \ell} y_{-\mathbf{k}, \ell}^{+} .
\end{aligned}
$$

En changeant $k$ en $-k$, on obtient :

$$
\begin{aligned}
H & =\sum_{\mathbf{k}, \ell=1, . ., L} E_{-\mathbf{k}, \ell} y_{-\mathbf{k}, \ell}^{+} y_{-\mathbf{k}, \ell}+E_{-\mathbf{k}, \ell+L} y_{\mathbf{k}, \ell} y_{\mathbf{k}, \ell}^{+} \\
& =\sum_{\mathbf{k}, \ell=1, . ., L} E_{-\mathbf{k}, \ell} y_{-\mathbf{k}, \ell} y_{-\mathbf{k}, \ell}^{+}+E_{-\mathbf{k}, \ell+L} y_{\mathbf{k}, \ell}^{+} y_{\mathbf{k}, \ell}
\end{aligned}
$$


ce qui montre que $E_{\mathbf{k}, \ell+L}=E_{-\mathbf{k}, \ell}$. La matrice $E_{\mathbf{k}}$ a donc une structure particulière :

$$
E_{\mathbf{k}}=\left(\begin{array}{cccccc}
E_{\mathbf{k}, 1} & & & & & \\
& . . & & & & \\
& & E_{\mathbf{k}, L} & & & \\
& & E_{-\mathbf{k}, 1} & & \\
& & & & . . & \\
& & & & & E_{-\mathbf{k}, L}
\end{array}\right)
$$

Pour construire la matrice de changement de base $P_{\mathbf{k}}$, on suit la méthode de Bogolubov, en introduisant les commutateurs $\left[X_{s}, X_{s^{\prime}}^{+}\right]$et $\left[Y_{s}, Y_{s^{\prime}}^{+}\right]$(l'indice $k$ est omis afin de rendre les formules plus lisibles). On pose :

$$
\begin{aligned}
{\left[X_{s}, X_{s^{\prime}}^{+}\right] } & =X_{s} X_{s^{\prime}}^{+}-X_{s^{\prime}}^{+} X_{s}=g_{s, s^{\prime}} \\
{\left[Y_{s}, Y_{s^{\prime}}^{+}\right] } & =g_{s, s^{\prime}}^{\prime}
\end{aligned}
$$

Or $\left[X_{s}, X_{s^{\prime}}^{+}\right]$peut encore s'écrire :

$$
\begin{aligned}
{\left[X_{s}, X_{s^{\prime}}^{+}\right] } & =\left[\sum_{\nu} P_{s, v} Y_{\nu}, \sum_{\mu} Y_{\mu}^{+}\left(P^{+}\right)_{\mu, s^{\prime}}\right] \\
& =\sum_{\nu, \mu}\left[P_{s, v} Y_{\nu} Y_{\mu}^{+}\left(P^{+}\right)_{\mu, s^{\prime}}-Y_{\mu}^{+}\left(P^{+}\right)_{\mu, s^{\prime}} P_{s, \nu} Y_{\nu}\right] \\
& =\sum_{\nu, \mu}\left[P_{s, v}\left(g_{v, \mu}^{\prime}+Y_{\mu}^{+} Y_{\nu}\right)\left(P^{+}\right)_{\mu, s^{\prime}}-Y_{\mu}^{+}\left(P^{+}\right)_{\mu, s^{\prime}} P_{s, v} Y_{\nu}\right] \\
& =\sum_{\nu, \mu} P_{s, \nu} g_{v, \mu}^{\prime}\left(P^{+}\right)_{\mu, s^{\prime}} .
\end{aligned}
$$

On en déduit $g=P_{\mathbf{k}} g^{\prime} P_{\mathbf{k}}^{+}$(relation d'orthogonalité) et compte tenu de $P_{\mathbf{k}}^{+} h_{\mathbf{k}} P_{\mathbf{k}}=E_{\mathbf{k}}$ :

$$
g h_{\mathbf{k}} P_{\mathbf{k}}=P_{\mathbf{k}} g^{\prime} E_{\mathbf{k}}
$$

Par définition, cette formule montre que la matrice $P_{\mathbf{k}}$ est la matrice des vecteurs propres de $g h_{\mathbf{k}}$ avec comme valeurs propres les $g^{\prime} E_{\mathbf{k}}$. Dans le cas présent, les opérateurs $b$ sont des bosons, de sorte que $g$ est une matrice diagonale par blocs qui s'écrit :

$$
g=\left(\begin{array}{ll}
I & \\
& -I
\end{array}\right) .
$$

Comme les $y_{\mathbf{k}, \ell}$ sont aussi des opérateurs bosons, $g^{\prime}=g$. Le problème consiste donc à chercher la matrice $P_{\mathbf{k}}$ et les énergies $E_{\mathbf{k}}$ solution de :

$$
\left(g h_{\mathbf{k}}\right) P_{\mathbf{k}}=P_{\mathbf{k}}\left(g E_{\mathbf{k}}\right)
$$

Dans le cas général, cette diagonalisation ne peut se faire que numériquement. On détermine ainsi $L$ valeurs propres indépendantes associées à une valeur de $\mathbf{k}$ donnée, ce qui correspond à $L$ branches d'ondes de spin. 


\subsection{Matrice de changement de base}

Examinons maintenant la structure de la matrice $P_{\mathbf{k}}$, et donc des vecteurs propres de $g h_{\mathbf{k}}$. La formule de changement de base donne :

$$
b_{\mathbf{k}, i}=\sum_{\ell=1, L} P_{\mathbf{k}, i, \ell} y_{\mathbf{k}, \ell}+\sum_{\ell=1, L} P_{\mathbf{k}, i, \ell+L} y_{-\mathbf{k}, \ell}^{+}
$$

et

$$
b_{-\mathbf{k}, i}^{+}=\sum_{\ell=1, L} P_{\mathbf{k}, i+L, \ell} y_{\mathbf{k}, \ell}+\sum_{\ell=1, L} P_{\mathbf{k}, i+L, \ell+L} y_{-\mathbf{k}, \ell}^{+} .
$$

En prenant l'adjoint de la première de ces deux relations et en changeant $k$ en $-k$, on obtient :

$$
b_{-\mathbf{k}, i}^{+}=\sum_{\ell=1, L} P_{-\mathbf{k}, i, \ell}^{*} y_{-\mathbf{k}, \ell}^{+}+\sum_{\ell=1, L} P_{-\mathbf{k}, i, \ell+L}^{*} y_{\mathbf{k}, \ell} .
$$

En identifiant avec la première équation, on constate que la matrice $P_{\mathbf{k}}$ possède elle aussi une structure par blocs :

$$
P_{\mathbf{k}}=\left(\begin{array}{cc}
U_{\mathbf{k}} & V_{-\mathbf{k}}^{*} \\
V_{\mathbf{k}} & U_{-\mathbf{k}}^{*}
\end{array}\right) \text { et } U_{\mathbf{k}, i, \ell}=P_{\mathbf{k}, i, \ell}, V_{\mathbf{k}, i, \ell}=P_{\mathbf{k}, i+L, \ell} .
$$

La relation entre les vecteurs $X_{\mathbf{k}}$ et $Y_{\mathbf{k}}$ peut donc se mettre sous la forme :

$$
\begin{aligned}
b_{\mathbf{k}, i} & =\sum_{\ell} U_{\mathbf{k}, i, \ell} y_{\mathbf{k}, \ell}+V_{-\mathbf{k}, i, \ell}^{*} y_{-\mathbf{k}, \ell}^{+}, \\
b_{-\mathbf{k}, i}^{+} & =\sum_{\ell} V_{\mathbf{k}, i, \ell} y_{\mathbf{k}, \ell}+U_{-\mathbf{k}, i, \ell}^{*} y_{-\mathbf{k}, \ell}^{+} .
\end{aligned}
$$

Remarque : si $M$ est une matrice d'éléments $M_{i, j}$, alors $M^{T}$ a pour éléments $M_{i, j}^{T}=M_{j, i}$ tandis que $M^{+}$a pour éléments $M_{i, j}^{+}=M_{j, i}^{*}$.

\subsection{Orthogonalité de la base des vecteurs propres}

Du fait de $g=P_{\mathbf{k}} g^{\prime} P_{\mathbf{k}}^{+}$, et dans le cas d'opérateurs du type boson, on trouve :

$$
\left(\begin{array}{cc}
I & \\
& -I
\end{array}\right)=\left(\begin{array}{cc}
U_{\mathbf{k}} & V_{-\mathbf{k}}^{*} \\
V_{\mathbf{k}} & U_{-\mathbf{k}}^{*}
\end{array}\right)\left(\begin{array}{cc}
I & \\
& -I
\end{array}\right)\left(\begin{array}{cc}
U_{\mathbf{k}}^{+} & V_{\mathbf{k}}^{+} \\
V_{-\mathbf{k}}^{T} & U_{-\mathbf{k}}^{T}
\end{array}\right)
$$

soit encore :

$$
\begin{aligned}
& I=U_{\mathbf{k}} U_{k}^{+}-V_{-\mathbf{k}}^{*} V_{-\mathbf{k}}^{T}, \\
& 0=U_{\mathbf{k}} V_{k}^{+}-V_{-\mathbf{k}}^{*} U_{-\mathbf{k}}^{T} .
\end{aligned}
$$

En développant les produits de matrice, on trouve finalement les deux conditions d'orthogonalité suivantes :

$$
\begin{aligned}
\delta_{i, j} & =\sum_{\ell} U_{\mathbf{k}, i, \ell} U_{\mathbf{k}, \ell, j}^{*}-V_{-\mathbf{k}, i, \ell}^{*} V_{-\mathbf{k}, j, \ell}, \\
0 & =\sum_{\ell} U_{\mathbf{k}, i, \ell} V_{\mathbf{k}, j, \ell}^{*}-V_{-\mathbf{k}, i, \ell}^{*} U_{-\mathbf{k}, j, \ell} .
\end{aligned}
$$




\subsection{Aimantation sur chaque site}

Pour mieux comprendre ce que représente physiquement une onde de spin, il est utile de calculer la valeur moyenne de $S_{m, i}^{3}$. On sait que par définition :

$$
S_{m, i}^{3}=S_{i}-b_{m, i}^{+} b_{m, i}
$$

et la quantité fondamentale est donc le nombre $b_{m, i}^{+} b_{m, i}$, qui représente l'écart ou la déviation par rapport à l'aimantation maximale possible (sur le site $i$ ) $S_{i}$ :

$$
\begin{aligned}
b_{m, i}^{+} b_{m, i} & =\frac{1}{N} \sum_{\mathbf{k}, \mathbf{k}^{\prime}} e^{i\left(\mathbf{k}-\mathbf{k}^{\prime}\right) \mathbf{R}_{m}} b_{\mathbf{k}, i}^{+} b_{\mathbf{k}^{\prime}, i} \\
& =\frac{1}{N} \sum_{\mathbf{k}, \mathbf{k}^{\prime}, \ell, \ell^{\prime}} e^{i\left(\mathbf{k}-\mathbf{k}^{\prime}\right) \mathbf{R}_{m}}\left(V_{-\mathbf{k}, i, \ell} y_{-\mathbf{k}, \ell}+U_{\mathbf{k}, i, \ell}^{*} y_{\mathbf{k}, \ell}^{+}\right)\left(U_{\mathbf{k}^{\prime}, i, \ell^{\prime}} y_{\mathbf{k}^{\prime}, \ell^{\prime}}+V_{-\mathbf{k}^{\prime}, i, \ell^{\prime}}^{*} y_{-\mathbf{k}^{\prime}, \ell^{\prime}}^{+}\right) .
\end{aligned}
$$

En prenant sa valeur moyenne, on fait apparaitre la distribution de Bose notée $n(E)=\frac{1}{e^{\hbar E / k_{B} T}-1}$. Il ne reste alors que :

$$
\left\langle b_{m, i}^{+} b_{m, i}\right\rangle=\sum_{\mathbf{k}, \ell} V_{-\mathbf{k}, i, \ell} V_{-\mathbf{k}, i, \ell}^{*}\left(1+n\left(E_{-\mathbf{k}, \ell}\right)\right)+U_{\mathbf{k}, i, \ell} U_{\mathbf{k}, i, \ell}^{*} n\left(E_{\mathbf{k}, \ell}\right)
$$

et donc :

$$
\left\langle S_{m, i}^{3}\right\rangle=S_{i}-\sum_{\mathbf{k}, \ell} V_{-\mathbf{k}, i, \ell} V_{-\mathbf{k}, i, \ell}^{*}\left(1+n\left(E_{-\mathbf{k}, \ell}\right)\right)+U_{\mathbf{k}, i, \ell} U_{\mathbf{k}, i, \ell}^{*} n\left(E_{\mathbf{k}, \ell}\right)
$$

Cette formule permet en premier lieu de s'assurer de l'auto-cohérence de l'approximation des ondes de spin : il faut en effet que la structure magnétique reste ordonnée et par conséquent que $\left\langle S_{m, i}^{3}\right\rangle$ garde une valeur finie au moins à température nulle. On remarque en particulier qu'il existe des fluctuations de point zéro qui réduisent la valeur de l'aimantation même à $T=0$ :

$$
\left\langle S_{m, i}^{3}(T=0)\right\rangle=S_{i}-\sum_{\mathbf{k}, \ell} V_{\mathbf{k}, i, \ell} V_{\mathbf{k}, i, \ell}^{*}
$$

A température finie, cette formule permet aussi de déterminer la température de transition, définie comme la température pour laquelle $\left\langle S_{m, i}^{3}\right\rangle=0$. Elle montre enfin que la population du mode propre d'énergie $E_{\mathbf{k}, \ell}$ contribue à accroître l'écart $S_{i}-\left\langle S_{m, i}^{3}\right\rangle$ avec un poids :

$$
V_{-\mathbf{k}, i, \ell} V_{-\mathbf{k}, i, \ell}^{*}+U_{\mathbf{k}, i, \ell} U_{\mathbf{k}, i, \ell}^{*}
$$

Une onde de spin est donc un état excité dans lequel le spin de chacun des ions de la maille tourne autour de sa direction privilégiée (excitation transverse) avec une même énergie (ou une même fréquence) $E_{\mathbf{k}, \ell}$. En revanche, pour un mode donné, l'écart par rapport à l'aimantation maximale $S_{i}$ n'est pas le même pour tous les ions $i$ du motif : certains spins seront faiblement déviés tandis que d'autres au contraire présenteront une déviation beaucoup plus forte. Cet effet tend à donner donner naissance à une "polarisation" des différents modes. Toutefois, il faut se garder de pousser l'analogie plus loin, et l'assimiler par exemple à la polarisation des modes de phonon. 


\section{CALCUL DE LA FONCTION DE DIFFUSION $S(Q, \omega)$}

Nous avons vu que la section efficace de diffusion des neutrons est proportionnelle à la fonction de diffusion :

$$
\mathcal{S}(\mathbf{Q}, \omega)=\sum_{a, b}\left(\delta_{a b}-\frac{Q_{a} Q_{b}}{Q^{2}}\right) S^{a b}(\mathbf{Q}, \omega)
$$

où les fonctions $S^{a b}(\mathbf{Q}, \omega)$ sont les fonctions de corrélation spin-spin :

$$
S^{\alpha \beta}(\mathbf{Q}, \omega)=\int d t e^{-i \omega t} \sum_{m, n, i, j} e^{i \mathbf{Q}\left(\mathbf{R}_{m}+\mathbf{u}_{i}-\mathbf{R}_{n}-\mathbf{u}_{j}\right)}\left\langle S_{m, i}^{\alpha} S_{n, j}^{\beta}(t)\right\rangle .
$$

Nous allons voir maintenant comment calculer cette quantité dans le cadre du formalisme développé plus haut. On obtient dans un premier temps :

$$
\begin{aligned}
\left\langle S_{m, i}^{\alpha} S_{n, j}^{\beta}(t)\right\rangle= & \frac{\sqrt{S_{i} S_{j}}}{2}\left\langle\left(b_{m, i}^{+} b_{m, i}\right)\left(\begin{array}{cc}
z_{i}^{\alpha} \bar{z}_{j}^{\beta} & z_{i}^{\alpha} z_{j}^{\beta} \\
\bar{z}_{i}^{\alpha} \bar{z}_{j}^{\beta} & \bar{z}_{i}^{\alpha} z_{j}^{\beta}
\end{array}\right)\left(\begin{array}{c}
b_{n, j}(t) \\
b_{n, j}^{+}(t)
\end{array}\right)\right\rangle \\
& +\eta_{i}^{\alpha} \eta_{j}^{\beta}\left(S^{2}-S\left\langle b_{m, i}^{+} b_{m, i}+b_{n, j}^{+} b_{n, j}\right\rangle\right) .
\end{aligned}
$$

Nous laisserons de côté, dans la toute la suite de cet exposé, le troisième terme de cette expression qui décrit en fait la partie statique de la fonction de corrélation. Il donne également une contribution dynamique, qui correspondent à des excitations (un continuum) longitudinales, mais son étude sort du cadre de ce cours. La suite du calcul fait alors intervenir différentes étapes que nous allons décrire pas à pas :

1. le facteur géométrique n'agissant que sur les indices $\alpha$ et $\beta$, on voit que son effet peut être pris en compte en remplaçant la matrice

$$
\left(\begin{array}{cc}
z_{i}^{\alpha} \bar{z}_{j}^{\beta} & z_{i}^{\alpha} z_{j}^{\beta} \\
\bar{z}_{i}^{\alpha} \bar{z}_{j}^{\beta} & \bar{z}_{i}^{\alpha} z_{j}^{\beta}
\end{array}\right)
$$

par :

$$
\left(\begin{array}{ccc}
\mathbf{z}_{i} M \overline{\mathbf{z}}_{j} & \mathbf{z}_{i} M \mathbf{z}_{j} \\
\overline{\mathbf{z}}_{i} M \overline{\mathbf{z}}_{j} & \overline{\mathbf{z}}_{i} M \mathbf{z}_{j}
\end{array}\right)
$$

où $M$ est une matrice définie par $M^{a b}=\delta_{a b}-\frac{Q_{a} Q_{b}}{Q^{2}}$.

2. On fait ensuite apparaitre les opérateurs "transformés" de Fourier :

$$
\begin{aligned}
\left\langle S_{m, i}^{\alpha} S_{n, j}^{\beta}(t)\right\rangle= & \frac{\sqrt{S_{i} S_{j}}}{2} \sum_{\mathbf{q}, \mathbf{q}^{\prime}} e^{i \mathbf{q} \mathbf{R}_{m}+i \mathbf{q}^{\prime} \mathbf{R}_{n}} \\
& \times\left\langle\left(b_{-\mathbf{q}, i}^{+} b_{\mathbf{q}, i}\right)\left(\begin{array}{ccc}
\mathbf{z}_{i} M \overline{\mathbf{z}}_{j} & \mathbf{z}_{i} M \mathbf{z}_{j} \\
\mathbf{z}_{i} M \overline{\mathbf{z}}_{j} & \overline{\mathbf{z}}_{i} M \mathbf{z}_{j}
\end{array}\right)\left(\begin{array}{c}
b_{\mathbf{q}^{\prime}, j}(t) \\
b_{-\mathbf{q}^{\prime}, j}^{+}(t)
\end{array}\right)\right\rangle
\end{aligned}
$$

3. puis les nouvelles variables $Y_{\mathbf{k}}$ :

$$
\begin{aligned}
& \left\langle S_{m, i}^{\alpha} S_{n, j}^{\beta}(t)\right\rangle=\frac{\sqrt{S_{i} S_{j}}}{2} \sum_{\mathbf{q}, \mathbf{q}^{\prime}, \ell, \ell^{\prime}} e^{i \mathbf{q} \mathbf{R}_{m}+i \mathbf{q}^{\prime} \mathbf{R}_{n}} \\
& \left\langle\left(y_{-\mathbf{q}, \ell}^{+} y_{\mathbf{q}, \ell}\right)\left(\begin{array}{cc}
U_{-\mathbf{q}, i, \ell}^{*} & V_{-\mathbf{q}, i, \ell}^{*} \\
V_{\mathbf{q}, i, \ell} & U_{\mathbf{q}, i, \ell}
\end{array}\right)\left(\begin{array}{cc}
\mathbf{z}_{i} M \overline{\mathbf{z}}_{j} & \mathbf{z}_{i} M \mathbf{z}_{j} \\
\overline{\mathbf{z}}_{i} M \overline{\mathbf{z}}_{j} & \overline{\mathbf{z}}_{i} M \mathbf{z}_{j}
\end{array}\right)\left(\begin{array}{cc}
U_{\mathbf{q}^{\prime}, j, \ell^{\prime}} & V_{-\mathbf{q}^{\prime}, j, \ell^{\prime}}^{*} \\
V_{\mathbf{q}^{\prime}, j, \ell^{\prime}} & U_{-\mathbf{q}^{\prime}, j, \ell^{\prime}}^{*}
\end{array}\right)\left(\begin{array}{c}
y_{\mathbf{q}^{\prime}, \ell^{\prime}}(t) \\
y_{-\mathbf{q}^{\prime}, \ell^{\prime}}^{\prime}(t)
\end{array}\right)\right\rangle .
\end{aligned}
$$


4. Compte tenu du fait que seules les valeurs moyennes suivantes sont non nulles :

$$
\begin{aligned}
& \left\langle y_{\mathbf{k}, \ell}^{+} y_{\mathbf{k}^{\prime}, \ell^{\prime}}(t)\right\rangle=\delta_{\mathbf{k}, k^{\prime}} \delta_{\ell, \ell^{\prime}} n\left(E_{\mathbf{k}, \ell}\right) e^{-i E_{\mathbf{k}, \ell t}} \\
& \left\langle y_{\mathbf{k}, \ell} y_{\mathbf{k}^{\prime}, \ell^{\prime}}^{+}(t)\right\rangle=\delta_{\mathbf{k}, k^{\prime}} \delta_{\ell, \ell^{\prime}}\left(1+n\left(E_{\mathbf{k}, \ell}\right)\right) e^{+i E_{\mathbf{k}, \ell} t}
\end{aligned}
$$

ne subsistent que les termes pour lesquels $\mathbf{q}^{\prime}=-\mathbf{q}, \ell=\ell^{\prime}$, correspondant aux éléments diagonaux du produit de matrice suivant :

$$
\left(\begin{array}{cc}
U_{-\mathbf{q}, i, \ell}^{*} & V_{-\mathbf{q}, i, \ell}^{*} \\
V_{\mathbf{q}, i, \ell} & U_{\mathbf{q}, i, \ell}
\end{array}\right)\left(\begin{array}{cccc}
\mathbf{z}_{i} M \overline{\mathbf{z}}_{j} & \mathbf{z}_{i} M \mathbf{z}_{j} \\
\overline{\mathbf{z}}_{i} M \overline{\mathbf{z}}_{j} & \overline{\mathbf{z}}_{i} M \mathbf{z}_{j}
\end{array}\right)\left(\begin{array}{cc}
U_{\mathbf{q}^{\prime}, j, \ell} & V_{-\mathbf{q}^{\prime}, j, \ell}^{*} \\
V_{\mathbf{q}^{\prime}, j, \ell} & U_{-\mathbf{q}^{\prime}, j, \ell}^{*}
\end{array}\right)
$$

soit pour le premier avec $\mathbf{k}=\mathbf{q}^{\prime}=-\mathbf{q}$ :

$$
A_{i, j, \ell}=\left(\begin{array}{ll}
U_{\mathbf{k}, i, \ell}^{*} V_{\mathbf{k}, i, \ell}^{*}
\end{array}\right)\left(\begin{array}{ccc}
\mathbf{z}_{i} M \overline{\mathbf{z}}_{j} & \mathbf{z}_{i} M \mathbf{z}_{j} \\
\overline{\mathbf{z}}_{i} M \overline{\mathbf{z}}_{j} & \overline{\mathbf{z}}_{i} M \mathbf{z}_{j}
\end{array}\right)\left(\begin{array}{c}
U_{\mathbf{k}, j, \ell} \\
V_{\mathbf{k}, j, \ell}
\end{array}\right)
$$

et pour le second avec $\mathbf{k}=-\mathbf{q}^{\prime}=\mathbf{q}$ :

$$
B_{i, j, \ell}=\left(\begin{array}{lll}
V_{\mathbf{k}, i, \ell} & U_{\mathbf{k}, i, \ell}
\end{array}\right)\left(\begin{array}{cccc}
\mathbf{z}_{i} & M \overline{\mathbf{z}}_{j} & \mathbf{z}_{i} M \mathbf{z}_{j} \\
\overline{\mathbf{z}}_{i} M \overline{\mathbf{z}}_{j} & \overline{\mathbf{z}}_{i} M \mathbf{z}_{j}
\end{array}\right)\left(\begin{array}{c}
V_{\mathbf{k}, j, \ell}^{*} \\
U_{\mathbf{k}, j, \ell}^{*}
\end{array}\right)
$$

de sorte que :

$$
\begin{aligned}
\left\langle S_{m, i}^{\alpha} S_{n, j}^{\beta}(t)\right\rangle= & \frac{\sqrt{S_{i} S_{j}}}{2} \sum_{\mathbf{k}, \ell}\left(e^{-i \mathbf{k}\left(\mathbf{R}_{m}-\mathbf{R}_{n}\right)} n\left(E_{\mathbf{k}, \ell}\right) e^{-i E_{\mathbf{k}, \ell} t} A_{i, j, \ell}(\mathbf{k})\right. \\
& \left.+e^{+i \mathbf{k}\left(\mathbf{R}_{m}-\mathbf{R}_{n}\right)}\left(1+n\left(E_{\mathbf{k}, \ell}\right)\right) e^{+i E_{\mathbf{k}, \ell} t} B_{i, j, \ell}(\mathbf{k})\right) .
\end{aligned}
$$

5. On utilise alors les résultats suivants

$$
\begin{aligned}
\int d t e^{-i \omega t} e^{i E t} & =\delta(\omega-E) \\
\sum_{m, n, \mathbf{k}} e^{i \mathbf{Q}\left(\mathbf{R}_{m}-\mathbf{R}_{n}\right)} e^{i \mathbf{k}\left(\mathbf{R}_{m}-\mathbf{R}_{n}\right)} & =\sum_{\mathbf{k}, \tau} \delta(\mathbf{Q}+\mathbf{k}-\tau)
\end{aligned}
$$

pour obtenir finalement :

$$
\begin{aligned}
\mathcal{S}(\mathbf{Q}, \omega)= & \sum_{\mathbf{k}, \tau} \delta(\mathbf{Q}+\mathbf{k}-\tau) \sum_{\ell} \\
& \times n\left(E_{-\mathbf{k}, \ell}\right) \delta\left(\omega+E_{-\mathbf{k}, \ell}\right)\left(\sum_{i, j} \frac{\sqrt{S_{i} S_{j}}}{2} e^{i \mathbf{Q}\left(\mathbf{u}_{i}-\mathbf{u}_{j}\right)} A_{i, j, \ell}(-\mathbf{k})\right) \\
& +\left(1+n\left(E_{\mathbf{k}, \ell}\right)\right) \delta\left(\omega-E_{\mathbf{k}, \ell}\right)\left(\sum_{i, j} \frac{\sqrt{S_{i} S_{j}}}{2} e^{i \mathbf{Q}\left(\mathbf{u}_{i}-\mathbf{u}_{j}\right)} B_{i, j, \ell}(\mathbf{k})\right)
\end{aligned}
$$

où $\tau$ est un vecteur du réseau réciproque associé au réseau magnétique. On pourra également vérifier que :

$$
B_{i, j, \ell}^{*}=A_{j, i, \ell} .
$$

Ces calculs permettent de démontrer qu'une expérience de diffusion inélastique des neutrons permet a priori de mesurer les énergies des $L$ branches de dispersion, associées aux $L$ branches d'ondes de spin du système. Le calcul de $\mathcal{S}(\mathbf{Q}, \omega)$ peut également être très utile pour prépaper les mesures, de manière 
à déterminer la zone de Brillouin (et donc le vecteur $\mathbf{Q}$ ) le plus favorable du point de vue de la section efficace.

\section{EXEMPLES D'ILLUSTRATION}

Pour finir, on se propose de traiter deux exemples simples.

\subsection{Le ferromagnétisme}

Dans le cas d'un système ferromagnétique avec un seul ion par maille et une interaction entre les $\zeta$ proches voisins, on obtient :

$$
\begin{aligned}
& \mathbf{z}_{1}=(1, i, 0), \\
& \eta_{1}=(0,0,1) \\
& J_{\mathbf{k}}=J \sum_{v} e^{i \mathbf{k} \Delta_{v}}
\end{aligned}
$$

et :

$$
\begin{aligned}
& \Omega_{\mathbf{k}}=S\left(\begin{array}{cc}
\zeta J & \\
& \zeta J
\end{array}\right) \\
& h_{\mathbf{k}}=-\Omega_{\mathbf{k}}+\frac{S}{2} J_{\mathbf{k}}\left(\begin{array}{cc}
2 & \\
& 2
\end{array}\right)=S\left(\begin{array}{ll}
-\zeta J+J_{\mathbf{k}} & \\
& -\zeta J+J_{\mathbf{k}}
\end{array}\right) .
\end{aligned}
$$

On obtient donc une unique branche d'onde de spin d'énergie :

$$
E_{\mathbf{k}}=S\left(-\zeta J+J_{\mathbf{k}}\right)
$$

et au voisinage de $\mathbf{k}=0$, la dispersion est parabolique :

$$
E_{\mathbf{k}} \approx k^{2} .
$$

Cette excitation est le mode de Goldstone de la phase ferromagnétique. Par ailleurs, on peut montrer que l'aimantation diverge toujours, à température finie, en dimensions 1 et 2 . Autrement dit, la phase ordonnée n'existe qu'en dimension 3. On montre enfin que la fonction de diffusion peut se mettre sous la forme :

$$
\begin{aligned}
\mathcal{S}(Q, \omega)= & \sum_{\mathbf{k}, \tau} \delta(\mathbf{Q}+\mathbf{k}-\tau) \\
& \times n\left(E_{-\mathbf{k}}\right) \delta\left(\omega+E_{-\mathbf{k}}\right) \frac{S}{2}\left(1+\frac{Q_{z}^{2}}{Q^{2}}\right)+\left(1+n\left(E_{\mathbf{k}}\right)\right) \delta\left(\omega-E_{\mathbf{k}}\right) \frac{S}{2}\left(1+\frac{Q_{z}^{2}}{Q^{2}}\right) .
\end{aligned}
$$

\subsection{L'antiferromagnétisme}

Dans le cas antiferromagnétique, on doit considérer un réseau avec deux ions par maille, le premier avec un spin dirigé selon $z$ et l'autre selon $-z$ :

$$
\begin{aligned}
& \mathbf{z}_{1}=(1, i, 0), \\
& \mathbf{g}_{1}=(0,0,1), \\
& \mathbf{z}_{2}=(-1, i, 0), \\
& \mathbf{g}_{2}=(0,0,-1) .
\end{aligned}
$$


Par ailleurs, on ne tient compte dans cet exemple que de l'interaction $J$ entre spins proches voisins (chaque spin a $\zeta$ proches voisins) :

$$
J_{\mathbf{k}, 1,2}=J \sum_{v} e^{i \mathbf{k} \Delta_{v}}=J_{\mathbf{k}}
$$

On obtient :

$$
\begin{aligned}
& \Omega_{\mathbf{k}}=S \zeta\left(\begin{array}{llll}
-J & & & \\
& -J & & \\
& & -J & \\
& & & -J
\end{array}\right) \\
& h_{\mathbf{k}}=-\Omega_{\mathbf{k}}+\frac{S}{2}\left(\begin{array}{llll} 
& -2 J_{\mathbf{k}}^{*} \\
-2 J_{\mathbf{k}}^{*} & -2 J_{\mathbf{k}} &
\end{array}\right) \\
& g h_{\mathbf{k}}=S\left(\begin{array}{cccc}
\zeta J & & & -J_{\mathbf{k}} \\
& \zeta J & -J_{\mathbf{k}}^{*} & \\
& J_{\mathbf{k}} & -\zeta J & \\
J_{\mathbf{k}}^{*} & & & -\zeta J
\end{array}\right) .
\end{aligned}
$$

Les énergies des ondes de spin sont donc solutions de :

$$
\begin{aligned}
\left(\zeta J S-E_{\mathbf{k}}\right)\left(-\zeta J S-E_{\mathbf{k}}\right)+S^{2}\left|J_{\mathbf{k}}\right|^{2} & =0 \\
E_{\mathbf{k}} & =S \sqrt{(\zeta J)^{2}-\left|J_{\mathbf{k}}\right|^{2}}
\end{aligned}
$$

avec au voisinage de $k=0$, une dispersion linéaire :

$$
E_{\mathbf{k}} \approx k \text {. }
$$

Là aussi, cette excitation est le mode de Goldstone de la phase magnétique. Encore une fois, l'aimantation diverge à température finie en dimensions 1 et 2 , montrant que la phase ordonnée n'existe qu'en dimension 3. Le cas antiferromagnétique est un cas interessant où la transformation de Bogolubov n'est pas une diagonalisation simple. L'état fondamental n'est pas l'état de Néel, et comporte à température nulle, des déviations du spin par rapport à l'état classique. Ces fluctuations de point zéro réduisent d'ailleurs l'energie de ce fondamental par rapport à celle de l'état de Néel.

La matrice de changement de base s'écrit :

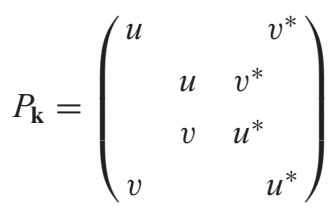

avec

$$
|u|^{2}=\frac{1}{2}\left(1+\frac{\zeta J S}{E_{\mathbf{k}}}\right) \quad \text { et } \quad|v|^{2}=\frac{1}{2}\left(-1+\frac{\zeta J S}{E_{\mathbf{k}}}\right) .
$$

Le calcul de la fonction de diffusion ne présente pas de difficultés particulières. Avec :

$$
\begin{aligned}
& A_{1,1,1}=u^{*} u \mathbf{z}_{1} M \mathbf{z}_{1} \\
& A_{1,1,2}=v^{*} v \overline{\mathbf{z}}_{1} M \mathbf{z}_{1}
\end{aligned}
$$




$$
\begin{aligned}
& A_{2,2,1}=v^{*} v \overline{\mathbf{z}}_{2} M \mathbf{z}_{2} \\
& A_{2,2,2}=u^{*} u \mathbf{z}_{2} M \overline{\mathbf{z}}_{2} \\
& A_{1,2,1}=u^{*} v \mathbf{z}_{1} M \mathbf{z}_{2} \\
& A_{1,2,2}=v^{*} u \overline{\mathbf{z}}_{1} M \overline{\mathbf{z}}_{2} \\
& A_{2,1,1}=v^{*} u \overline{\mathbf{z}}_{2} M \overline{\mathbf{z}}_{1} \\
& A_{2,1,2}=u^{*} v \mathbf{z}_{2} M \mathbf{z}_{1}
\end{aligned}
$$

on trouve :

$$
\begin{aligned}
\mathcal{S}(\mathbf{Q}, \omega)= & \sum_{\mathbf{k}, \tau} \delta(\mathbf{Q}+\mathbf{k}-\tau) \\
& \times n\left(E_{-\mathbf{k}}\right) \delta\left(\omega+E_{-\mathbf{k}}\right)\left(\frac{S}{2} A(-\mathbf{k})\right)+\left(1+n\left(E_{\mathbf{k}}\right)\right) \delta\left(\omega-E_{\mathbf{k}}\right)\left(\frac{S}{2} A(\mathbf{k})\right), \\
A(k)= & \left(1+\frac{Q_{z}^{2}}{Q^{2}}\right)\left(2\left(u^{*} u+v^{*} v\right)-\left(u^{*} v+u v^{*}\right)\left(e^{i \mathbf{k}\left(\mathbf{u}_{1}-\mathbf{u}_{2}\right)}+e^{-i \mathbf{Q}\left(\mathbf{u}_{1}-\mathbf{u}_{2}\right)}\right)\right) \\
= & 2\left(1+\frac{Q_{z}^{2}}{Q^{2}}\right) \frac{\zeta J S-\left|J_{\mathbf{k}}\right| S \cos \left(\mathbf{Q}\left(\mathbf{u}_{1}-\mathbf{u}_{2}\right)\right)}{E_{\mathbf{k}}} .
\end{aligned}
$$

On constate que la fonction de diffusion est d'autant plus intense que le vecteur $\mathbf{Q}$ est proche du vecteur antiferromagnétique défini par $\mathbf{Q}\left(\mathbf{u}_{1}-\mathbf{u}_{2}\right)=\pi$. En revanche, $\mathcal{S}(\mathbf{Q}, \omega)$ s'annule pour $\mathbf{Q}=0$. Le facteur géométrique en $\left(1+\frac{Q_{z}^{2}}{Q^{2}}\right)$ illustre bien le fait que l'on ne mesure que les composantes de spin perpendiculaires au vecteur d'onde $\mathbf{Q}$.

\section{CONCLUSION}

Quelles conlusions tirer de ces calculs formels ? Tout d'abord, nous avons vu que le modèle des ondes de spin suppose l'existence d'une structure ordonnée, et qu'il n'est valable que dans la limite des basses températures ou d'un spin classique.

Dans ce cas, le modèle prévoit l'existence de $L$ branches d'excitations (de type boson) où $L$ est le nombre d'atomes dans la maille magnétique. Leur dispersion est donnée par les $E_{\mathbf{k}, \ell}$. Sauf cas particulier, seul le calcul numérique permet de les obtenir.

Ces excitations sont en outre associées à des mouvements de précession des spins autour de leur direction moyenne, et qui se propagent dans le cristal. On dit souvent qu'il s'agit d'excitations transverses, car le spin tourne dans le plan perpendiculaire à cette direction privilégiée.

Pour s'assurer de la stabilité de la structure magnétique, nous avons vu qu'il fallait calculer l'aimantation moyenne du système sur chaque site. En fonction du système étudié, on prendra donc soin de vérifier que $\left\langle S_{m, i}^{3}\right\rangle$ garde une valeur finie.

Nous avons également établi l'expression de la fonction de diffusion, qui est directement proportionnelle à la section efficace de diffusion des neutrons. 


\section{BIBLIOGRAPHIE}

Quelques références générales sur la théorie des ondes de spin :

\section{Références}

[1] P.W. Anderson, Phys. Rev. 83, 1260 (1951)

[2] R. Kubo, Phys. Rev. 87, 568 (1952)

[3] T. Oguchi, Phys. Rev. 117, 117 (1960)

[4] C. Tsallis, Diagonalization method for the general Hamiltonian of an assembly of bosons, J. Math. Phys., 19, 277 (1978)

[5] D.C. Mattis, Theory of Magnetism I, Springer Verlag, 1988

[6] R.M. White, Quantum Theory of Magnetism, Springer Verlag, 1987

[7] A. Auerbach, Interacting electrons and Quantum Magnetism, Springer Verlag, 1994 Izyumov, Plenum 1994 